\title{
L'ordre des Évangiles : une nouvelle mise à jour
}

Par

\section{Pierre-Maurice Bogaert}

Université catholique de Louvain, Abbaye de Maredsous

$\mathrm{S}$

i Bruce M. Metzger n'est pas le premier à s'être intéressé à la séquence des Évangiles Theodor Zahn a rassemblé et interprété vers la fin du XIX ${ }^{\mathrm{e}}$ siècle une documentation très étendue ${ }^{1}-$, il a le mérite d'avoir proposé aux biblistes une liste commode des ordres attestés à sa connaissance ${ }^{2}$. Il y a une vingtaine d'années, j'avais essayé de rajeunir cet inventaire et, dans un second temps, de le mettre en relation avec les ordres des Vivants dans l'Apocalypse $(4,7)$ et dans Ézéchiel $(1,10 \text { et } 10,14)^{3}$. Depuis lors, mon attention a été attirée sur des témoins qui avaient échappé et sur d'autres nouvellement découverts, principalement dans le domaine latin ${ }^{4}$. Dans cet hommage à l'éditeur d'Esther, de Marc et de Daniel dans la Vetus Latina de Beuron, auteur de divers travaux sur la critique textuelle de l'Ancien Testament et du Nouveau, je voudrais mettre à jour l'inventaire proposé en 1999, et il y a de bonnes raisons de le faire. Il est possible aujourd'hui de mieux saisir le mouvement vers l'ordre des Évangiles devenu universel. La combinaison avec les ordres des Vivants et avec les systèmes d'identification Évangélistes-Vivants n'interviendra qu'en fonction de l'ordre des Évangiles.

\footnotetext{
${ }^{1}$ ZAHN 1892.

2 MetZGer 1997, p. 296-297.

${ }^{3}$ Bogaert 1999 et Bogaert 2001.

${ }^{4}$ Voir ci-dessous n. 89.
} 
Pour la clarté, je rappelle d'emblée la liste des ordres attestés (Tableau I), les listes des Vivants (Tableau II) et les systèmes de correspondance Évangiles-Vivants d'après Theodor Zahn (Tableau III) ${ }^{5}$. La présente mise à jour ne recourra qu'accessoirement à ces combinaisons, car elle vise à reconnaître de préférence les ordres des Évangiles attestés par des listes, par des codices ou suivis par des commentaires.

\begin{tabular}{|l|l|l|l|}
\hline & Metzger & \multicolumn{1}{|c|}{ Ordre des Évangiles } & \\
\hline E1 & $a$ & $\mathrm{Mt}-\mathrm{Mc}-\mathrm{Lc}-\mathrm{Jn}$ & grec, latin, syriaque, etc. \\
\hline E2 & $d$ & $\mathrm{Mt}-\mathrm{Mc}-\mathrm{Jn}-\mathrm{Lc}$ & latin, syriaque \\
\hline E3 & $e$ & $\mathrm{Mt}-\mathrm{Lc}-\mathrm{Mc}-\mathrm{Jn}$ & grec, latin, copte \\
\hline E4 & $c$ & $\mathrm{Mt}-\mathrm{Jn}-\mathrm{Mc}-\mathrm{Lc}$ & grec, latin, syriaque \\
\hline E5 & $b$ & $\mathrm{Mt}-\mathrm{Jn}-\mathrm{Lc}-\mathrm{Mc}$ & grec, latin, gothique \\
\hline E6 & - & $(\mathrm{Jn}-\mathrm{Lc})-\mathrm{Mc}-\mathrm{Mt}$ & latin \\
\hline E7 & $f$ & $\mathrm{Jn}-\mathrm{Mt}-\mathrm{Mc}-\mathrm{Lc}$ & copte, arménien \\
\hline E8 & $g$ & $\mathrm{Jn}-\mathrm{Mt}-\mathrm{Lc}-\mathrm{Mc}$ & grec, latin \\
\hline E9 & - & $\mathrm{Jn}-\mathrm{Lc}-\mathrm{Mt}-\mathrm{Mc}$ & grec \\
\hline E10 & $h$ & $\mathrm{Mc}-\mathrm{Mt}-\mathrm{Lc}-\mathrm{Jn}$ & anglo-saxon (latin) \\
\hline E11 & $i$ & $\mathrm{Mc}-\mathrm{Lc}-\mathrm{Mt}-\mathrm{Jn}$ & anglo-saxon (latin) \\
\hline E12 & - & $(\mathrm{Lc}-) \mathrm{Jn}-\mathrm{Mc}$ & copte \\
\hline E13 & - & $\mathrm{Jn}-\mathrm{Mc}-\mathrm{Mt}-(\mathrm{Lc})$ & latin \\
\hline
\end{tabular}

Tableau I. Ordre des Évangiles

La première colonne reprend la numérotation proposée en 1999, E1 à E126 , avec l'addition de E13. La deuxième colonne rappelle le sigle de B.M. Metzger.

La dernière colonne signale les langues dans lesquelles sont attestées les séquences.

\begin{tabular}{|l|l|}
\hline V1 : Apocalypse 4,7 & $\mathrm{L}-\mathrm{T}-\mathrm{H}-\mathrm{A}$ \\
\hline V2 : Apocalypse 4,7 variante latine & $\mathrm{L}-\mathrm{H}-\mathrm{T}-\mathrm{A}$ \\
\hline V3 : Ézéchiel 1,10 LXX et TM & $\mathrm{H}-\mathrm{L}-\mathrm{T}-\mathrm{A}$ \\
\hline V4 : Ézéchiel 10,14 hébreu et Vulgate & $\mathrm{T}-\mathrm{H}-\mathrm{L}-\mathrm{A}$ \\
\hline V5 : Ézéchiel 10,14? - Apocalypse 4,7 & $\mathrm{T}-\mathrm{L}-\mathrm{H}-\mathrm{A}$ \\
\hline V6 : Ezéchiel $1,10-$ Apocalypse 4,7 & $\mathrm{H}-\mathrm{T}-\mathrm{L}-\mathrm{A}$ \\
\hline
\end{tabular}

Tableau II. Ordre des Vivants

Lion (L) - Taureau (T) - Homme (H) - Aigle (A)

${ }^{5}$ ZAHN 1883, p. 258-262.

${ }^{6}$ De E1 à E7, la liste se conforme à celle de ZAHN 1890-1892, p. 364-375, 1013-1015. 
L'ordre V2 des Vivants selon l'Apocalypse est attesté chez Victorin de Poetovio, dans le Traité pseudo-cyprianique De centesima (PS-CY cent 27) ${ }^{7}$ et dans le Liber Mozarabicus Sacramentorum $^{8}$. Les ordres V5 et V6 sont attestés dans deux sermons apparentés De Salomone attribuables à Grégoire d'Elvire (CPL 555 ; CPPM I B, 5027 ) 9. Dans toutes ces séquence, l'Aigle vient en finale.

Nous rencontrerons aussi ci-dessous les séquences $\mathrm{H}-\mathrm{L}-\mathrm{A}-\mathrm{T}$ en $\mathrm{E} 2, \mathrm{H}-\mathrm{T}-\mathrm{A}-\mathrm{L}$ en $\mathrm{E} 3$, et $\mathrm{H}-\mathrm{T}-\mathrm{L}-\mathrm{A}$ en E8. Elles ne sont pas explicables par le texte biblique ; elles sont les résultantes respectivement de l'application d'un système d'identification (Tableau III) à un ordre codicologique (Tableau I).

\begin{tabular}{|l|l|l|l|l|l|}
\hline & S I & \multicolumn{1}{|c|}{ S II } & \multicolumn{1}{c|}{ S III } & \multicolumn{1}{|c|}{ S IV } & \multicolumn{1}{c|}{ S V } \\
\hline Matthieu & H & H & L & H & L \\
\hline Marc & L & A & H & T & T \\
\hline Luc & T & T & T & L & H \\
\hline Jean & A & L & A & A & A \\
\hline
\end{tabular}

Tableau III. Systèmes d'identification évangélistes - vivants ${ }^{10}$ Lion (L) - Taureau (T) - Homme (H) - Aigle (A)

Le présent exposé ne porte pas sur l'identification des Quatre Vivants. Toutefois il est utile de rappeler les témoins des différents systèmes, car il y a moins d'hésitation sur ce point que sur l'ordre. Inutile d'énumérer les nombreux témoins de S I $(\text { Marc }=\text { lion })^{11}$. Les témoins de S II (Marc = aigle) sont Irénée, Victorin de Poetovio, Fortunatien, Chromace d'Aquilée, Augustin (qui connaît S III), un poème attribué à Juvencus, le Book of Durrow ${ }^{12}$ et, chez les Byzantins, André de Césarée et Anastase le Sinaïte qui dépendent d'Irénée. Les témoins de S III (Marc $=$ homme) sont Hippolyte ${ }^{13}$, Augustin $^{14}$, qui le préfère à S II, et les auteurs qui dépendent d'Augustin, Primasius et Bède le Vénérable. Les témoins de S IV (Marc =taureau ; Luc $=$ lion) sont des textes grecs de date et d'authenticité difficiles à établir ${ }^{15}$. Chez les Latins, Berengaud (vers 900 ou au XII ${ }^{\mathrm{e}}$ s.) atteste S V (Marc = taureau; Luc = homme), mais il sait que S I est utilisé par les antiquiores ${ }^{16}$.

\footnotetext{
${ }^{7}$ PLS I,61.

${ }^{8}$ FÉROTIN 1912, col. 743 (le texte n'est pas cité in extenso) ; voir GRYSON 2000-2003, p. 15 et 257 (c'est le ms. VL 271); BOGAERT 2001, p. 469.

${ }^{9}$ Voir d'une part CC SL 69, p. 255 pour V5 et, pour V6, la nouvelle édition de Delmulle 2021, surtout p. 256 (ms. P) et 257-258 (ms. G).

${ }^{10}$ D'après ZAHN 1883, p. 258-262.

${ }^{11} \mathrm{~S}$ I est associé à un ordre rare des évangiles (E10) dans la refonte par Jérôme du commentaire de Victorin sur l'Apocalypse (CC SL 5, p. 151, 153, 155 ; ed. GRYSON 2017) et à E2 chez « Théophile » en Gaule vers 500. Voir ci-dessous.

12 Pour le détail des nombreuses attestations de S I, S II et S III, voir BogAERT 2001, p. 460-461. Fortunatien (ci-dessous E3) et le Book of Durrow (ci-dessous E5) viennent ici en plus pour S II.

${ }^{13}$ BOGAERT 2001, p. 466-468.

${ }^{14}$ Augustin, De cons. ev. 1,6 (9) (CSEL 43, p. 9). Augustin y présente S III comme probabilius aliquid; on le retrouve dans son Tract. in Joh. 35 (CC SL 36, p. 326-327) où il suit l'ordre V1 des Vivants.

15 La Synopsis scripturae sacrae athanasienne, CPG, n 2249 (PG 28,431), et un prologue attribué à Épiphane, dans SODEN 1902, p. 303-304.

${ }^{16}$ PL 17,802-803 sous le nom d'Ambroise. Sur ce Berengaudus, voir Jullien, Perelman 1994, p. $233-236$.
} 


\section{L'ordre devenu commun : E1}

E1 (a) : Mt $-M c-L c-J n$

L'ordre Mt - Mc - Lc - Jn est de loin le plus répandu, si bien qu'il est vain de vouloir énumérer tous ses représentants. Il faut cependant rappeler son histoire. Irénée est le premier à proposer la succession chronologique des quatre Évangiles dans cet ordre. Il accorde une importance capitale au corpus des quatre Évangiles, mais il n'en a pas un ordre arrêté ${ }^{17}$. Le Canon de Muratori (Mc? - Lc - Jn), qui est la traduction latine au IV siècle d'un original grec $^{18}$, atteste vraisemblablement le même ordre, de type chronologique.

En grec, E1 est l'ordre des grands onciaux et celui de nombreuses listes canoniques et d'innombrables manuscrits. Ceux-ci représentent la descendance de cet ordre depuis les grands scriptoria impériaux, Alexandrie, Césarée, Antioche et finalement Constantinople. Le codex de papyrus Bodmer XIV-XV $\left(\mathrm{P}^{75}\right)$ vers 200 et un codex bilingue gréco-copte sur parchemin (0139) ${ }^{19}, \mathrm{du} \mathrm{V} \mathrm{V}^{\mathrm{e}}-\mathrm{VI}^{\mathrm{e}}$ siècle, donnent $\mathrm{Lc}-\mathrm{Jn}$ et pourraient être complémentaires d'un autre donnant $\mathrm{Mt}-\mathrm{Mc}$; ils seraient ainsi les précurseurs de l'ordre reçu, mais, à défaut de Mt - Mc, ce n'est qu'une hypothèse. Le perfectionnement du codex a permis le regroupement des quatre Évangiles et ensuite la fixation de son ordre, au moins dans le domaine grec.

Dans le domaine latin, c'est presque certainement la nouvelle traduction de Jérôme (383) qui a introduit l'ordre devenu commun ${ }^{20}$. Elle est accompagnée de la synopse des Canons d'Eusèbe qui met en évidence les passages semblables des quatre Évangiles. Jérôme s'en explique longuement dans son prologue Nouum opus ${ }^{21}$. Comme B.M. Metzger l'a justement noté, les canons eusébiens largement diffusés ont assuré le succès de l'ordre E1 dont ils ont paru inséparables ${ }^{22}$. Nous verrons cependant, à propos d'un témoin grec de E5, que les canons d'Eusèbe était applicables à un autre ordre $^{23}$. Le plus ancien témoin de la version hiéronymienne des Évangiles (Saint-Gall, Stiftsbibliothek 1395, sigle : S), qu'on peut dater tôt dans $\mathrm{le}^{\mathrm{e}}$ siècle, donc encore du vivant de Jérôme, donne déjà l'indication des sections eusébiennes en marge ${ }^{24}$.

Toujours dans le domaine latin, Augustin connaît et utilise l'ordre E1, puisqu'il connaît la traduction de Jérôme, mais nous verrons que Jérôme lui-même et Augustin connaissent aussi et utilisent l'ordre E3.

En syriaque, le Sinaiticus des Évangiles séparés (IV $-V^{\mathrm{e}}$ siècle) et le nouveau témoin palimpseste découvert par Sebastian Brock ( $\mathrm{VI}^{\mathrm{e}}$ siècle $)^{25}$ ont l'ordre E1, mais le ms. Cureton a l'ordre E2 (voir ci-dessous). Le témoignage d'Éphrem est à écarter ${ }^{26}$; il utilise le Diatessaron.

\footnotetext{
${ }^{17}$ GUIGNARD 2017 dit parfaitement la position d'Irénée qui ne s'en tient pas à un ordre fixe.

${ }^{18}$ Houghton 2016, p. 17; Guignard 2019.

${ }^{19}$ VAN HAELST $1976, \mathrm{n}^{\circ} 409$.

${ }^{20}$ Houghton 2016, p. 32.

${ }^{21}$ Biblia Sacra $1994^{4}$, p. 1515-1526.

22 MetzGer 1997, p. 296 ; voir aussi Regul 1969, p. 214.

${ }^{23}$ CRAWFORD 2018 ; voir ci-dessous à E5.

24 TURNER 1931.

${ }^{25}$ BROCK 2016 : La succession Lc - Jn est assurée par un bifolio ; la succession Mt - Mc est assurée par celle des cahiers IV et $\mathrm{V}$.
} 
Je n'ai rencontré aucun témoin manuscrit de la série complète E1 qui soit du premier tiers $\mathrm{du} \mathrm{IV}^{\mathrm{e}}$ siècle ou antérieur ${ }^{27}$.

\section{Autres ordres commençant par Matthieu : E2, E3, E4, E5}

$E 2(d): M t-M c-J n-L c$

En grec, apparemment cet ordre n'est pas attesté. Th. Zahn a raison d'écarter le témoignage d'Origène ${ }^{28}$.

En latin, cet ordre est celui d'un des deux témoins de la Stichométrie de Mommsen (olim : Cheltenham, Phillipps 12266 ; Rome, Bibl. Naz. Centr. Vitt. Emm. II, Vitt. Emm. 1325, f. $39 \mathrm{v}-40)^{29}$. Il me paraît moins assuré - je veux dire moins ancien - que celui du codex de Saint-Gall (ci-dessous E4).

C'est aussi l'ordre d'un commentaire des Évangiles souvent attribué à un certain Théophile, mais anonyme et rédigé vers 500 en Gaule; il faut évidemment suivre les manuscrits, prologue et texte, d'après l'édition de Michael M. Gorman ${ }^{30}$, et non les éditions anciennes qui ont introduit l'ordre habituel ${ }^{31}$. Manifestement, l'ordre E2 est contraignant pour "Théophile », car, dans le prologue, il y soumet l'identification commune des Vivants (S I) : $\mathrm{Mt}=$ homme $; \mathrm{Mc}=$ lion $; \mathrm{Jn}=$ aigle $; \mathrm{Lc}=$ taureau : la suite $\mathrm{H}-\mathrm{L}-\mathrm{A}-\mathrm{T}$ n'est pas autrement attestée et n'est pas biblique (voir ci-dessus Tableau II).

E2 est encore l'ordre de la recension II de l'Expositio IV Evangeliorum du Pseudo-Jérôme souvent attribuée à Grégoire le Grand (la recension I a l'ordre E4) ${ }^{32}$.

E2 est vraisemblablement l'ordre attesté par un poème d'Avit de Vienne (De consolatoria castitatis laude) dont l'énumération des Vivants correspond à E4 si l'on suit le système S II ou plutôt et mieux à E2 si l'on suit le système $\mathrm{S} I$ devenu commun de son temps ${ }^{33}$.

En syriaque, cet ordre est attesté par le ms. Cureton, vetus syra des Évangiles séparés (Londres, BL, Add. $14451 ; \mathrm{V}^{\mathrm{e}} \mathrm{s}$.).

\section{$E 3(e): M t-L c-M c-J n$}

En grec, on ne peut mentionner qu'un témoin isolé de la Liste grecque des 60 livres canoniques (Londres, $B L$, Add. 17469 , fol. 1v) ${ }^{34}$. Si Irénée connaissait déjà un ordre matériel des Évangiles, ce serait plutôt E3 que E5 ${ }^{35}$.

\footnotetext{
${ }^{26}$ Il d'agit d'un complément non éphrémien au Commentaire sur le Diatessaron arménien ; voir SC 121, p. 409.

27 Je pense au Sinaiticus et au Vaticanus. - Regul 1969, p. 215, fait observer que le Carmen aduersus Marcionitas, du Pseudo-Tertullien, II,61 (CC SL 2, p. 1428) énumère les évangélistes dans l'ordre E1 et ne peut être très ancien ; aujourd'hui on le date des années 420-450, peut-être en Gaule (CPL 36).

${ }^{28}$ ZAHN 1890-1892, p. 373, n. 1.

${ }^{29}$ PREUSCHEN 1893, p. 139-141.

${ }^{30}$ GORMAN 2003. - La qualité de «plus ancien » ne convient plus après la publication du commentaire de Fortunatianus.

31 PLS III, 1283.

32 BischOFF 1954, p. 236-237.

${ }^{33}$ HeCQUeT-Noti 2011, p. 163-166 (lignes 398-401).
} 
En latin, au cours du IV siècle, il faut mentionner deux noms importants, l'Ambrosiaster ${ }^{36}$ et Fortunatianus d'Aquilée. Le témoignage de Fortunatien est nouveau ; on le doit à l'identification de son commentaire par Lukas J. Dorfbauer ${ }^{37}$. La séquence E3 est contraignante pour le commentateur, car l'ordre de ses identifications, homme - taureau - aigle - lion, n'est attesté ni dans Ézéchiel ni dans l'Apocalypse et ne peut donc intervenir ${ }^{38}$.

Occasionnellement Jérôme, dans le commentaire de Marc et dans l'Adversus Iovinianum (393), et Augustin, In Ioh. XXXVI,5, utilisent cet ordre, ce qui conduit à tenir E3 pour un ordre dominant chez les Latins avant la nouvelle traduction de Jérôme. Par deux fois, en effet, ce dernier cite les Évangiles dans l'ordre E3 et y conforme les identifications qu'il préfère, celles du système devenu commun, $\mathrm{S} \mathrm{I}: \mathrm{Mt}=$ homme, $\mathrm{Lc}=$ taureau, $\mathrm{Jn}=$ aigle, $\mathrm{Mc}=\operatorname{lion}^{39}$. (L'ordre des Vivants $\mathrm{H}-\mathrm{T}-\mathrm{A}-\mathrm{L}$ n'est attesté ni dans Ézéchiel ni dans l'Apocalypse.) Augustin de son côté, dans un texte daté de juillet-octobre 414 énumère les Vivants dans l'ordre d'Apc 4,7 (S III) et leur fait correspondre l'ordre E3 des évangélistes ${ }^{40}$.

Une version latine du Concile de Laodicée, qui donne principalement l'ordre E1 et accessoirement l'ordre E4, présente cependant dans le ms. П (Paris, BnF, lat. 3848A, début IX ${ }^{\mathrm{e}} \mathrm{s}$., provenance Troyes et Pithou), une séquence incomplète Mt - Lc, le quaternion suivant étant $\operatorname{perdu}^{41}$. Cette séquence ne peut guère être que $\mathrm{E} 3$.

En copte (akhmîmique), trois feuillets avec Lc 12-13 et Lc 17-18 appartenaient à un codex d'un seul cahier, du IV ${ }^{\mathrm{e}}$ ou du $\mathrm{V}^{\mathrm{e}}$ siècle, comportant à l'origine, selon $\mathrm{L}$. Th. Lefort, $\mathrm{Mt}-\mathrm{Lc}-$ Jn sans Mc. Son raisonnement suppose en effet que Mt précède Lc puisque les feuillets de Lc appartiennent à la deuxième moitié du codex et que la place disponible (176 pages) correspond de façon exacte à $\mathrm{Mt}$ et au début de $\mathrm{Lc}^{42}$. La succession $\mathrm{Mt}-\mathrm{Lc}$ se retrouvera aussi en E8.

\section{$E 4(c): M t-J n-M c-L c$}

En grec, cet ordre est attesté par le minuscule 888 (Venise, Marciana, gr. 26 ; XIV $-\mathrm{XV}^{\mathrm{e}} \mathrm{s}$.). On le rencontre chez Jean Chrysostome (In Rom 1,1 ; PG 60, 395 et 397), mais peu après, dans le même passage, on lit la séquence Mt - Lc - Mc qui est E3. Le codex grec 0234 du $\mathrm{VIII}^{\mathrm{e}}$ siècle, s'il contenait les quatre Évangiles ; commençait par $\mathrm{Mt}$ - Jn, ce qui donne le choix

\footnotetext{
${ }^{34}$ ZAHN, 1890-1892, t. II, p. 289, n. 1 ; GREGORY 1900-1909, p. 196 ; voir aussi MeRCATI 1941, p. 59, 82-84 (sans mention de l'ordre rare).

${ }^{35}$ Irénée, Adv. Haer. III,9-11 ; III,11,7 ; IV,6,1. CAMPENHAUSEN 1968, p. 228-229 et 242 ; SKEAT 1992, p. 194-196. Skeat a raison de dire qu'Ez 1,10 est sous-jacent à l'explication qu'Irénée donne d'Ap 4,7 en $A d v$. Haer. III,11,8, mais rien n'oblige à penser que sa source ignorait Ap 4,10 et suivait déjà E5, dont en cette place il s'écarterait : Irénée est inconstant dans son énumération des Évangiles : GUIGNARD 2017 (ci-dessus, n. 17).

${ }^{36}$ Ambrosiaster, Quaestiones Veteris et Novi Testamenti 4 (CSEL 430,19-22 ; ed. A. SOUTER) ; voir REGUL 1969, p. 218.

${ }^{37}$ DorfBAuER 2017, p. 109 (Regula), p. 135-142 (Capitula), p. 116 (Mt), 227 (Lc), 233 (Jn). On retrouve ici l'ancien usage : cata Matheum (ligne 717), cata Lucanum (ligne 731), cata Marcum (ligne 753).

38 BASTIT 2017.

39 Hieronymus, Adversus Iouinianum I,26 (PL 23,259), ce traité date de 393 ; Id., Tractatus in Marci Euangelium 1 (CC SL 78, p. 452).

${ }^{40}$ Augustinus, In Iohannis Euangelium XXXVI,5 (CC SL 36, p. 326-327).

41 TURNER 1939, p. 391.

42 LEFORT 1953, p. 17-20, pl. 1 ; LEFORT 1949.
} 
entre E4 et E5. Christian Duthmar, de Stavelot, mort vers 880, écrit qu'il a vu deux livres des Évangiles grecs, dits de saint Hilaire, avec Matthieu et Jean en tête ${ }^{43}$. Puisqu'il ne précise pas l'ordre des deux autres Évangiles, il faut le présumer courant, ce qui fait pencher la balance pour E4. La même question se pose à propos de Palladius qui, face à Cyrille d'Alexandrie, a dans les mains un $\Delta \dot{\varepsilon} \lambda \tau \imath$ v contenant Matthieu et Jean, donc E4 ou E5 ${ }^{44}$.

En latin, E4 est attesté dans des listes vénérables, dans la Stichométrie de Mommsen selon le manuscrit de Saint Gall, Stiftsbibl. $133^{45}$, et dans le canon du Claromontanus des Épîtres pauliniennes (Paris, $B n F$, gr. $107+107 \mathrm{~A}+107 \mathrm{~B}$ ) du VI ${ }^{\mathrm{e}} \mathrm{s}$; sud de l'Italie ${ }^{46}$.

Un manuscrit de la version latine du Concile de Laodicée (Paris, BnF, lat. 1451, VIII ${ }^{\mathrm{e}}-\mathrm{IX}^{\mathrm{e}}$ s.; prov. Saint-Maur-des-Fossés), citée déjà en E3, donne l'ordre E4 ${ }^{47}$. C'est une lectio difficilior qu'on ne peut négliger.

La plupart des manuscrits de la recension I de l'Expositio du Pseudo-Jérôme sur les Évangiles ont l'ordre E4 ${ }^{48}$.

J'ai rattaché de préférence à E2 le témoignage d'Avit de Vienne.

En syriaque, il faut mentionner des témoins anciens des Évangiles (Peshitta) conservés à la British Library ${ }^{49}$ que Theodor Zahn rattachait à l'ordre E5. Il faut être plus réservé, je crois. La séquence Mt - Jn est assurée dans le ms. 89 (Add. 12141, du VI ${ }^{\mathrm{e}}-\mathrm{VII}^{\mathrm{e}}$ siècle) et dans le ms. 96 (Add. 17115, du VI ${ }^{\mathrm{e}}$ s.). Par ailleurs, je ne vois pas de témoin sûr de la succession Lc - Mc. Je rattacherais donc ces deux témoins à E4. Il n'y a rien à tirer de certain pour les mss 97 et 98 du catalogue de W. Wright. Il reste que la grande majorité des témoins de la Peshitta ont l'ordre E1.

\section{E5 (b) : Mt - Jn - Lc - Mc, « ordre occidental »}

Cet ordre est parfois appelé « occidental ». En grec, il faut signaler un codex de papyrus du $\mathrm{III}^{\mathrm{e}}$ s., P. Chester Beatty I + P. Vindob. G.31974 (0104; P45) ${ }^{50}$; un codex de parchemin, vers 400, Washington, Freer Gallery of Art 06.274 (032, Freer III) ${ }^{51}$. La jonction de deux fragments, 073 et 084, permet de reconnaître l'ordre E5 ; il portait les sections eusébiennes en marge ${ }^{52}$. Dans le célèbre Codex Bezae, bilingue, l'ordre a pu être conditionné par l'usage latin, mais il y a assez d'exemples grecs pour se dispenser de cette explication. Il faut ajouter trois mss en minuscule qui ont en commun que Mt et Jn sont accompagnés du commentaire de Jean Chrysostome : Munich, $U B, 2^{\circ}$ cod. 30 (sigle X) - après reconstitution de l'ordre des cahiers $^{53}-$, Paris, $B n F$, gr. 200, du X $\mathrm{X}^{\mathrm{e}}-\mathrm{XI}^{\mathrm{e}}$ s., incomplet (sigle 308), et Paris, $B n F$, gr. $201 \mathrm{du}$ $\mathrm{X}^{\mathrm{e}}-\mathrm{XI}^{\mathrm{e}}$ s. (sigle 055 ou 309). S'ajoute à cela le témoignage de Grégoire de Nazianze. Dans le

\footnotetext{
${ }^{43}$ PL 106, 1266.

${ }^{44}$ PG 68,133 (De adoratione).

${ }^{45}$ PREUSCHEN 1893, p. 139-141.

${ }^{46}$ FREDE 1964, p. 25-26.

47 TURNER 1939, p. 391.

${ }^{48}$ GRIESSER 1937, p. 281.

49 WRIGHT 1870.

50 SKEAT 1993.

51 VAN HAELST 1976, n 331.

52 CRAWFORD 2018.

${ }^{53}$ ZAHN 1890-1892, t. II, p. 370, n. 1.
} 
poème De veris Scripturae libris, celui-ci semble énumérer les évangélistes dans l'ordre devenu commun E1 ${ }^{54}$. Mais Gregory a fait observer que le ms. 536 du Nouveau Testament grec, autrefois Londres, Highgate, Burdett-Coutts II,7, maintenant Ann Arbor, University of Michigan, Ms. 24, du XII ${ }^{\mathrm{e}}$ siècle, donne les poèmes sur les miracles du Christ dans l'ordre E5 ${ }^{55}$. En réalité, les éditions anciennes que j'ai consultées (Bâle 1550, $2^{\mathrm{e}}$ partie, p. 38-39; Jacques de Billy, rééd. Paris, t. II, 1611, p. 101-103) donnent aussi l'ordre E5 pour les poèmes sur les miracles et les paraboles ${ }^{56}$. En attendant une édition critique, on peut tenir que l'accord du ms. 536 avec les éditions anciennes de Grégoire de Nazianze atteste une ordonnance antique. Les Mauristes, suivis par Migne, n'indiquent pas de source manuscrite pour le nouvel ordonnancement des poèmes ${ }^{57}$.

En latin, la majorité des Évangiles non vulgates ont l'ordre E5 :

- a (VL 3): Vercellensis (Italie, IV ${ }^{\mathrm{e}} \mathrm{s}$.) ;

- b (VL 4) : Veronensis (Vérone, fin $\mathrm{V}^{\mathrm{e}} \mathrm{s}$.) ;

- d (VL 5) : Codex Bezae Cantabrigiensis (Lyon ?, Beirouth ?, Ve s.);

- e (VL 2) : Palatinus (Trente ?, V $\mathrm{V}^{\mathrm{e}} \mathrm{s}$.) ;

- $\mathrm{f}(\mathrm{VL} 10)$ : Brixianus (Italie du N., $\left.1 / 2 \mathrm{VI}^{\mathrm{e}} \mathrm{s}.\right)$;

- ff2 (VL 8) : Corbeiensis (Italie, $\mathrm{V}^{\mathrm{e}} \mathrm{s}$.) ;

- n (VL 16) : Sangallensis (Italie, $\mathrm{V}^{\mathrm{e}} \mathrm{s}$.);

- q (VL 13) : Monacensis (Italie du N. ou Illyrie, VIe-VIIe s.).

Présents dans de nombreux manuscrits des Évangiles vulgates, les prologues dits monarchiens supposent et suivent 1'ordre E5 ; on leur donne souvent une origine priscillianiste ${ }^{58}$. Toujours en latin, le Liber de divinis scripturis qui cite longuement la Bible à livre ouvert d'après des témoins de la vetus latina, donne les Évangiles dans l'ordre E5. La séquence Mt Jn - Lc est ferme ${ }^{59}$. Quand Mc est cité (une seule fois) il suit Luc ${ }^{60}$. Le témoignage de Chromace d'Aquilée doit être évalué, car son ordre des Évangiles est conditionné par la référence à Ez 1,10 (V3) et par son système de correspondance entre évangélistes et Vivants (S II) ; toutefois à cette date, vers 400 , et dans cette région, la séquence E5 explique au mieux sa présentation accordant $\mathrm{V} 3$ et $\mathrm{S} \mathrm{II}^{61}$. Un fragment latin, attribué par le manuscrit, non identifié, à Polycarpe et attribué à un Pacatus, qui pourrait être l'ami de Paulin de Nole, donne explicitement l'ordre $\mathrm{E}^{62}$.

\footnotetext{
${ }^{54}$ PG 37,474.

${ }^{55}$ GREGORY 1900-1909, p. 855.

56 PG 37,487-496.

${ }^{57}$ Mon regretté collègue Justin Mossay m'a assisté jadis de sa compétence en ce domaine qui lui tenait à cœur.

${ }^{58}$ Regul 1969, p. 218 ; Houghton 2016, p. 197.

${ }^{59}$ DE BRUYNE 1931, p. 128.

${ }^{60}$ CSEL 12, p. 381.

${ }^{61}$ CC SL 9A, p. 187-189.

${ }^{62}$ PG 5,1025-1026 ; HARNACK 1921, p. 270 (inc. Rationabiliter euangelistae ... ; expl. ad eandem patriam peruenitur). - Ce texte et quatre autres ont été été publiés la première fois par Feuardent dans son édition d'Irénée (1595/6) qui le tirait d'une catena dans un manuscrit non retrouvé de Verdun. Harnack n'a pas été suivi dans son attribution au rhéteur Latinius Drepanus Pacatus. L'attribution restera discutable aussi longtemps que les travaux en cours sur les compilations de Jean Diacre, en particulier l'Expositum sur l'heptateuque (CPL951)
} 
On rattachera à E5 un bas-relief du $\mathrm{IV}^{\mathrm{e}} \mathrm{s}$. trouvé à Spolète, aujourd'hui à Rome, représentant une barque. Jésus, à la proue, est à droite ; de gauche à droite rament les évangélistes Matthieu (perdu), Jean, Luc et Marc ${ }^{63}$.

De même, la séquence des Vivants dans le Book of Durrow (Dublin, Trinity College 57 ; $2 / 2 \mathrm{VII}^{\mathrm{e}}$ s. ; Northumbrie ?), le plus ancien manuscrit insulaire complet vulgate des quatre Évangiles, selon l'ordre commun (E1) donne successivement les symboles de l'aigle en tête de Marc, du taureau en tête de Luc et du lion en tête de Jean en conformité avec S II, système bien attesté et conforme à la combinaison $\mathrm{E} 5 / \mathrm{V} 3^{64}$.

En langue gotique, le célèbre et unique codex argenteus de la version dite d'Ulfilas $\left(\mathrm{V}^{\mathrm{e}}-\mathrm{VI}^{\mathrm{e}}\right.$ s.) donne l'ordre E5, preuve s'il en fallait encore que la version gotique des Évangiles, faite sur le grec, ne dépend pas du « texte byzantin » ${ }^{65}$.

Il est donc permis de qualifier E5 d'ordre occidental; sa prépondérance chez les Latins avant Jérôme et E1 est massive, bien que non exclusive (E2, E3, E4, E6, E8). Toutefois cet ordre est attesté anciennement en grec, au moins en Égypte ; il a été la source de la séquence gotique.

Dans le doute, on 1'a vu, j'ai rattaché de préférence à E4 plutôt qu'à E5 des Évangiles syriaques et le témoignage de Druthmar de Stavelot sur deux exemplaires grecs.

\section{Ordres commençant par Jean : (E6), E7, E8, E966}

E6 (-) : $(J n-L c)-M c-M t$

En latin, les parties conservées d'un codex des Évangiles d'origine vraisemblablement africaine (le texte transmis est certainement africain) sont aujourd'hui à Turin et proviennent de Bobbio (k, VL 1). Elles ont été éditées par J. Wordsworth, W. Sanday et H.J. White en $1886^{67}$. Dans l'introduction Wordsworth peut montrer par les signatures des cahiers que le codex avait contenu les quatre Évangiles et qu'il s'achevait avec $\mathrm{Mc}-\mathrm{Mt}$. Comme il n'y a pas d'exemple d'ordre commençant par Luc, il propose la séquence $\mathrm{Jn}-\mathrm{Lc}-\mathrm{Mc}-\mathrm{Mt}^{68}$. On en conclura que, vers 400 environ, en Afrique vraisemblablement, se transmettait un codex des quatre Évangiles dans un ordre particulier.

$E 7(f): J n-M t-M c-L c$

Cet ordre se rencontre dans les scalae coptes, qui sont des sortes de lexiques bibliques suivant l'ordre du texte. Dans les scalae, qui datent du XIII et du XIV ${ }^{\mathrm{e}}$ s., Jn vient en tête " à cause de sa facilité pour encourager l'étudiant» ou encore parce qu'il y a dans les trois

n'auront pas apporté une explication globale. Après Anne-Marie Genevois, Jérémy Delmulle en a repris l'étude et prépare une édition (DELMULLE 2018).

${ }^{63}$ STUhlFAUth 1942, p. 123 (l'auteur énumère les évangélistes de droite à gauche) ; DACL VII,2, ill. 6218 ; GARRUCCI 1872-1880, t. V, p. 138, pl. 395,6 ; CIL XI,4965 ; DIEHL 1961, nº 1965a note.

${ }^{64}$ Powell 1956, p. 16-21 : Mc (f. 84/76), Lc (f. 124/116, Jn (f. 191/173); Houghton 2016, p. 206.

${ }^{65}$ GRYSON 1990, p. 6.

${ }^{66}$ Voir aussi E13 ci-dessous, dans les ordres particuliers.

${ }^{67}$ WORDSWORTH 1886, p. XII.

${ }^{68}$ WORDSWORTH 1886 (ibid.) cite le ms. X, de Munich, mais ce doit être une erreur : voir ci-dessus E5. 
Évangiles synoptiques des mots qui ne sont pas l'Évangile de St Jean ${ }^{69} »$. Une des scalae les mieux ordonnées, Paris, $B n F$, copte 44 , a été publiée ${ }^{70}$.

L'ordre E7 est attesté aussi en arménien dans une liste des livres canoniques copiée au XIII ${ }^{\mathrm{e}}$ siècle par Mxit'ar Ayrivanec'i, d'après le vardapet Sarkavag. La source est grecque, proche de la « Liste des 60 livres $^{71} »$, mais adaptée aux usages arméniens ${ }^{72}$.

$E 8(g): J n-M t-L c-M c$

En grec, il faut mentionner la Synopsis de Jean-Chrysostome (CPG 559), le manuscrit grec minuscule 19, Paris, BnF, gr. 189, du XII ${ }^{\mathrm{e}}$ s., sans oublier le témoignage de l'Anthologie Palatine 1,80-85, signalé par B.M. Metzger ${ }^{73}$.

En latin, Tertullien regroupe Jean et Matthieu, apostoli, puis Luc et Marc, apostolici. Sans qu'on puisse conclure avec certitude qu'il lisait cet ordre dans un codex, il lui donne cependant une justification ${ }^{74}$.

Ce pourrait être aussi l'ordre de Victorin de Poetovio (avant 304) dans son Commentaire de l'Apocalypse avant la révision de Jérôme ${ }^{75}$. Victorin suit le système $\mathrm{S}$ II comme Irénée, ce qui supposerait un ordre $\mathrm{Mt}-\mathrm{Lc}-\mathrm{Jn}-\mathrm{Mc}$, non attesté par ailleurs. Dans le commentaire, à l'exception du lemme qui a l'ordre habituel d'Apc 4,7, Victorin suit deux fois successivement une variante inattendue : lion ( Jn) - homme (Mt) - taureau (Lc) - aigle (Mc); il suit l'ordre V2 (Apoc 4,7) qui, conjugué avec S II, donnerait l'ordre E8 ${ }^{76}$. Si Victorin a connu un ordre préalable, ce doit être E8. Dans le De fabrica mundi, 3, Victorin énumère les Vivants dans l'ordre homme - taureau - lion - aigle, ordre non attesté dans la Bible, sans préciser leur identification avec tel Évangile ${ }^{77}$; ce pourrait être le système S I appliqué à E3, mais il est peu vraisemblable que Victorin ait changé son système d'identification. En fin de compte, il n'est pas certain que, pour Victorin, un quelconque ordre des Évangiles (E3 ?, E8) constitue un donné préalable.

Il faut mentionner ici le sermon De Salomone, attribuable à Grégoire d'Elvire, qui dans une de ses deux formes propose la séquence rare $\mathrm{H}-\mathrm{T}-\mathrm{L}-\mathrm{A}$ (V6), sans évoquer les évangélistes. Cet ordre des Vivants met Grégoire dans le sillage de Victorin ${ }^{78}$.

\footnotetext{
${ }^{69}$ MALLON 1906-1907, p. 121 ; p. 235.

${ }^{70}$ MUNIER 1930, p. (1) - (26).

${ }^{71}$ Voir ci-dessus E3.

${ }^{72}$ ZAHN 1893, p. 148-150; STONE 1976 n'a édité que la liste des livres de l'Ancien Testament et des apocryphes. Les précisions viennent de mon collègue arménisant Bernard Coulie qui m'a très aimablement communiqué la bibliographie récente : SzEKULA 1949 et BAIS 2017, p. 80-83 (rien sur l'ordre des Évangiles).

${ }^{73}$ WALTZ 1928, p. 34-35.

${ }^{74}$ Tertullien, $A d v$. Marcionem IV,2,1 (SC 456, p. 68 ; ed. Cl. MoresChini, R. BraUn) ; PetitMengin 2002, p. 334.

${ }^{75}$ CC SL 5, p. 152-154 (GRYSON 2017).

${ }^{76}$ GRYSON 2017, p. 150-155.

77 Dulaey 1997, p. 140-141 et 217 ; GRYSON 2017, p. 296.

${ }^{78}$ Delmulle 2021 vient de l'étudier et de l'éditer (ci-dessus, n. 9). L'autre forme (CC SL 69, p. 255 ou PL 17,695B) a l'ordre T - L - H - A, qu'il est difficile d'expliquer : le taureau ne peut être que Luc, qui ne se trouve jamais en tête des séries connues, ou Marc qui vient en tête dans deux séries anglo-saxonnes atypiques (E10 et E11). Le Chérub (taureau ?) vient en tête des Vivants dans la liste d'Ez 10,14, propre à l'hébreu et donc à la Vulgate, absente dans la LXX et la vetus latina.
} 
Les Expositiunculae d'Arnobe le Jeune ( $\mathrm{V}^{\mathrm{e}} \mathrm{s}$.) sur les Évangiles traitent successivement de Jean, de Matthieu et de Luc $^{79}$. Parmi les ordres attestés, on ne peut penser qu'à E7, oriental, et à E8; nous optons pour E8.

$$
\text { E9 (-) : Jn }-\mathrm{Lc}-\mathrm{Mt}-\mathrm{Mc}
$$

Un seul manuscrit grec du XVI ${ }^{\mathrm{e}}$ s., Amsterdam, $U B$, Remonstr. 145, atteste cet ordre ${ }^{80}$. Il est celui que suit Irénée pour les Évangiles mis en relation avec le Vivants selon Ap 4,10 Ce n'est pas le seul ordre des Évangiles attesté chez Irénée, et celui-ci paraît conditionné par les correspondances qui sont les siennes (S II) ; il n'a probablement pas une origine codicologique et ne dépend pas d'une liste préalable.

\section{Ordres particuliers ou rares (E10, E11, E12, E13)}

$E 10(h): M c-M t-L c-J n$

Cet ordre particulier n'est attesté que dans un codex anglo-saxon des Évangiles, Londres, $B L$, Royal 1 A. xiv (Cantorbéry, Christ Church, $2 / 2 \mathrm{XII}^{\mathrm{e}} \mathrm{s}$. ${ }^{82}$. B.M. Metzger fait intervenir aussi la célèbre mosaïque du mausolée de Galla Placidia à Ravenne qui représente l'armoire aux Évangiles ( $\mathrm{V}^{\mathrm{e}} \mathrm{s}$.). Les quatre codices se présentent de la manière suivante :

$$
\begin{array}{lr}
\text { MARCVS } & \text { LVCAS } \\
\text { MATTEVS } & \text { IOANNES }
\end{array}
$$

Il ne semble pas que l'on puisse inférer une quelconque séquence certaine à partir de cette disposition. L'ordre E10 résulte de la refonte par Jérôme du commentaire de Victorin ${ }^{83}$. Il n'est donc pas un ordre codicologique.

E11 (i) : Mc $-L c-M t-J n$

Cet ordre, non moins curieux que le précédent, est attesté également dans un manuscrit anglo-saxon des Évangiles, Oxford, Bodleian Library, Hatton 38 (4090) (Cantorbéry ?, XII ${ }^{\mathrm{e}-}$ $\mathrm{XIII}^{\mathrm{e}}$ s. $)^{84}$. Comme le ms. Hatton 38 est une copie du Royal 1 A. xiv, on pourrait croire qu'il l'a trouvé ainsi disposé. Dans ces manuscrits chaque Évangile est copié séparément, chacun commençant avec un cahier, si bien que leur ordre peut être modifié à volonté. L'âge de la reliure pourrait fournir une indication.

$E 12(-)(L c)-J n-M c$

H. Quecke a décrit et édité un codex sahidique des Évangiles sur parchemin datable du V s. La succession Jn - Mc est certaine ; Lc - Jn - Mc est très vraisemblable, mais il n'y a pas

${ }^{79}$ CC SL 25A, p. 267-305 ; ed. K.D. DAUR. - Mon collègue Roger Gryson a attiré mon attention sur Arnobe le Jeune.

${ }^{80}$ GREGORY 1900-1909, p. 149 ; voir GUIGNARD 2017, p. 108.

${ }^{81}$ Irénée, Adv. Haer. III,11,8 (SC 211, p. 164-169 ; ed. A. RouSSEAU - L. DOUTRELEAU).

82 KER 1957, p. 315-316.

${ }^{83}$ CC SL 5, p. 151, 153, 155 (ed. R. GRYSON 2017).

${ }^{84}$ KER 1957, p. 386-387. 
de place pour Matthieu dans la reliure telle qu'elle est conservée ${ }^{85}$. La séquence $\mathrm{Lc}-\mathrm{Jn}-\mathrm{Mc}$ n'est pas autrement attestée ; la séquence Jn - Mc ne s'est trouvée qu'en E4.

$$
E 13(-) J n-M c-M t-(L c)
$$

Une fresque de la catacombe de $\mathrm{S}$. Gennaro à Naples représente la défunte, Bitalia, entre les livres des Évangiles : IOANNIS - MARCUS - le visage de la défunte en position d'orante MATTEU(S) - $(\text { LUCAS })^{86}$. On ne peut en inférer un ordre certain des Évangiles.

\section{Constatations finales}

On l'aura vu, l'utilisation des éditions anciennes doit toujours être contrôlée, car les exemples ne manquent pas de l'alignement d'un ordre devenu rare sur l'ordre dominant (E1). Ce qui est vrai des éditions, l'est aussi des lemmes, ajoutés ou corrigés après coup.

Il importe de lire les Pères avec des yeux non prévenus, attentifs aux imprévus. On a distingué, autant que faire se peut, les séquences matériellement assurées, codicologiquement significatives, et les énumérations qui peuvent suivre d'autres principes, telle la succession chronologique supposée des évangélistes.

Irénée et Tertullien nous assurent que dans les dernières décennies du $\mathrm{II}^{\mathrm{e}}$ siècle, la grande Église reconnaissait un Évangile en quatre, un «tétraévangile ». Autre chose en est la présentation matérielle. Certaines observations faites ci-dessus donnent à penser que, dans la progressive évolution du codex, le groupement de deux Évangiles et aussi de deux fois deux Évangiles a précédé le regroupement des quatre. Deux paires se distinguent : Mt - Jn (E4, E5, E11) ou Jn - Mt (E7, E8) d'une part et Lc - Mc (E5, E8) ou Mc - Lc (E4, E7, E11); Tertullien en donne une justification, qui n'est pas nécessairement la cause : Matthieu et Jean sont des apôtres, Luc et Marc sont venus ensuite (voir ci-dessus E8). Je note, d'un point de vue codicologique que le couple Luc - Marc associe le plus long et le plus court, Matthieu et Jean étant intermédiaires. L'ordre perçu comme chronologique, E1, est devenu dominant à partir des grands scriptoria grecs et de la diffusion des bibles en un volume.

Dans l'état de notre documentation, c'est surtout le monde latin qui atteste de multiples ordres : il reçoit tardivement, avec Jérôme, l'ordre E1, mais tout au long du $\mathrm{V}^{\mathrm{e}}$ siècle, on observe la persistance des ordres antérieurs: E2, E3, E4, E5 (appelé parfois «ordre occidental »), E6, E8. Jérôme lui-même a utilisé E3. Une telle multiplicité tient en partie à l'abondance de notre documentation latine, manuscrits et commentaires. Mais il faut aussi remonter plus haut. Dans les Églises latines, ce sont des textes grecs qui ont longtemps servi de modèle aux traducteurs, puis servi de critère aux réviseurs. Il est donc permis de croire que les ordres latins reflètent la variété de modèles grecs en partie perdus, ou plus tôt et systématiquement alignés.

Qu'en est-il de l'Afrique latine ? L'ordre E4, Mt - Jn - Mc - Lc, de la stichométrie de Mommsen selon le meilleur témoin, est africain et peut-être donatiste (vers 350); on le retrouve dans le canon du Claromontanus, bilingue des Épîtres pauliniennes copié au milieu $\mathrm{du} \mathrm{V}^{\mathrm{e}}$ siècle, vraisemblablement dans le sud de l'Italie. L'ordre E6, (Jn - Lc) - Mc - Mt, est attesté par un ms. venu d'Afrique, le Bobbiensis. Si les listes des conciles africains, de la fin

\footnotetext{
${ }^{85}$ QUECKE 1984, p. 7-9.

${ }^{86}$ GARRUCCI 1872-1880, t. II, pl. 99,1 ; DIEHL 1961, n 1965a note ; CIL X,1521 ; DACL XII,1, col. 770.
} 
$\mathrm{du} \mathrm{IV}^{\mathrm{e}}$ siècle, donnent l'ordre E1, on a vu qu'Augustin se réfère occasionnellement à l'ordre E3.

En Italie, vers 350, l'ordre E3 est attesté fermement par 1'Ambrosiaster et Fortunatien d'Aquilée, mais ensuite E5 devient dominant. L'ordre E5, connu très tôt en grec, est attesté abondamment chez les Latins et par la version gotique dépendant du grec, indice qui pointe vers la présence de cet ordre dit « occidental » à Constantinople ou dans son environnement, avant E1. Faut-il envisager aussi une influence grecque sur le Nord de l'Italie - par l'Illyrie ? Ce serait une preuve que les formes dominantes de texte et d'ordre, E1, diffusées par quelques grands scriptoria grecs et vraisemblablement Constantinople n'ont pas gagné instantanément la périphérie. L'ordre E5 est progressivement supplanté par E1, c'est-à-dire par la révision de Jérôme (Vulgate).

Le syriaque aussi atteste la variété : E2 et E4 à côté de E1; toutefois le prestige du Diatessaron modifiait les données en retardant la décision. Il faut être aussi aux accords latinsyriaque (E2, E4).

D'autres réflexions sont permises, moins assurées. Les épîtres pauliniennes, groupées ou non par destinataire, ont été rangées en fonction de la longueur ${ }^{87}$. Si ce principe avait été appliqué aux Évangiles, en grec comme en latin, l'ordre résultant serait Lc - Mt - Jn - Mc ; cet ordre n'est pas attesté et l'argument de la longueur ne semble pas avoir joué. Si, comme il est vraisemblable, les deux œuvres lucaniennes, l'Évangile et les Actes des Apôtres, ont circulé, réunies ensemble avant que l'Évangile ne rejoigne le tétraévangile, se pourrait-il que les séquences terminant par Luc en gardent le souvenir : E2 latin et syriaque, E4 grec, latin et syriaque $^{88}$ ? Les Évangiles ont dû circuler d'abord séparément l'un de l'autre. Notre documentation ne permet guère de le montrer, sauf tardivement dans des Évangiles anglo-saxons qui tout en groupant les quatre Évangiles laissent la possibilité de les utiliser indépendamment l'un de l'autre (E10, E11).

Le présent inventaire est une révision et une mise à jour ; il pourra être encore complété. Non seulement des cas d'ordre divergeant en grec et dans les versions, surtout orientales, ont pu nous échapper, mais encore de nouvelles découvertes et de nouvelles éditions permettront vraisemblablement de perfectionner la présente liste et d'enrichir ses conclusions ${ }^{89}$.

\footnotetext{
${ }^{87}$ FREDE 1966-1971, p. 290-303; voir p. 292.

88 BOGAERT 2006, p. 528-529.

${ }^{89}$ À côté des mises à jour de détail, voici en bref les principales additions : E1 : le troisième témoin de la vetus syra publié par S. BROCK 2016; E2 : le ms. Phillipps de la stichométrie de Mommsen, aujourdhui : Rome, Bibl. Naz. Vitt. Emm. 1325 ; Avit de Vienne, éd. HeCQUET-Noti 2011 ; la recension II de l'Expositio euangeliorum du Pseudo-Jérôme; E3 : Fortunatianus, éd. DORFBAUER 2017 ; citations occasionnelles d'Augustin; concile de Laodicée latin, ms. П ; E4 : recension I de l'Expositio euangeliorum du Pseudo-Jérôme ; concile de Laodicée latin, ms. F; E5 : le papyrus grec 073 + 084, éd. CRAWFORD 2018 ; les prologues dit monarchiens, édités par REgul 1969 ; le Pseudo-Polycarpe (Pacatus ?) ; E7 : arménien (précisions) ; E8 : Tertullien, Grégoire d'Elvire (J. Delmulle, 2021).
} 


\section{BIBLIOGRAPHIE}

BAIS, Marco, 2017 : « Canone biblico armeno », dans E.G. FerRUGIA, Emidio Vergani (éd.), Il canone biblico nelle Chiese orientali. Atti del simposio Pontificio Istituto Orientale, Roma 23 marzo 2010 (Orientalia Christiana Analecta, 302), Roma, Pontificio Istituto Orientale, p. 69-94.

Biblia Sacra 19944 : Biblia Sacra iuxta Vulgatam Versionem, Stuttgart, Deutsche Bibelgesellschaft.

BisCHOFF, Bernard, 1954 : «Wendepunkte in der Geschichte der lateinischen Exegese im Frühmittelalter », dans Sacris Erudiri 6, p. 189-279.

BASTIT, Agnès, 2017 : «Le prologue aux commentarii sur les évangiles de Fortunatien. Étude analytique et enquête sur les sources », dans Fortunatianus redivivus. Bischof Fortunatian von Aquileia und sein Evangelienkommentar. Hrsg. von L.J. DORFBAUER unter Mitarbeit von V. ZIMMERL-PANAGL, Berlin, Boston, W. de Gruyter, p. 1-46.

BOGAERT, Pierre-Maurice, 1999 : «Ordres anciens des évangiles et tétraévangile en un seul codex », Revue théologique de Louvain 30, 297-314.

- 2001 : «Les Quatre Vivants, l'Évangile et les évangiles », Revue théologique de Louvain $32,457-478$.

— 2006 : « Les bibles d'Augustin », Revue théologique de Louvain 37, p. 513-531.

Brock, Sebastian, 2016 : «Two Hitherto Unattested Passages of the Old Syriac Gospels in

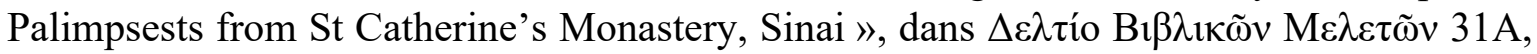
p. 7-18.

CAmPenhausen, Hans von, 1968: Die Entstehung der christlichen Bibel (Beiträge zur historischen Theologie, 30), Tübingen, J.C.B. Mohr (P. Siebeck).

Crawford, Matthew, 2018 : "A new witness to the 'western' ordering of the Gospels GA 073+084 », The Journal of Theological Studies, NS 69/2, p. 477-483.

DE BRUYNE, Donatien, 1931 : «Étude sur le Liber de diuinis scripturis », Revue bénédictine 43, p. 124-141.

Delmulle, Jérémy, 2018 : «Un fantôme et un pseudonyme (?). 'Catellus', Iohannes Diaconus et l'auteur du Liber Promissionum et Praedictionum Dei », Revue bénédictine 128, p. 5-30.

— 2021 : «Un tractatus sur Prou. 30, 15-20 (CPPM I 5027) et la question de son attribution à Grégoire d'Elvire ", dans M. Pignot (éd.), Latin Anonymous Sermons from Late Antiquity and the Early Middle Ages (AD 300-800) : Classification, Transmission, Dating (Instrumenta Patristica et Mediaevalia, 86 ; Ministerium Sermonis, 4), Turnhout, Brepols, p. 207264.

DIEHL, Ernestus, $1961^{2}$ : Inscriptiones Latinae Christianae Veteres, I-III, Berlin, Weidmann.

Dorfbauer, Lukas J., 2017 : Fortunatianus Aquileiensis, Commentarii in in Evangelia. Ediert von Lukas J. DORFBAUER (CSEL 103), Berlin, Boston, W. de Gruyter.

Dulaey, Martine, 1997 : Victorin de Poetovio, Sur l'Apocalypse suivi du Fragment chronologique et de La construction du monde. Introduction, texte critique, traduction, commentaire et index, par Martine DulaEY (SC 423), Paris, Cerf. 
FÉROTIN, Marius, 1912 : Le Liber Mozarabicus Sacramentorum et les manuscrits mozarabes (Monumenta Ecclesiae Liturgica, 6), Paris, Firmin-Didot.

FREDE, Hermann Josef, 1964 : Altlateinische Paulus-Handschriften (Aus der Geschichte der lateinischen Bibel 4), Freiburg, Herder.

- 1966-1971 : Epistulae ad Philippenses et ad Colossenses (Vetus Latina 24/2) ed. Hermann Josef FREDE, Freiburg Br.

GARRUCCI, Raffaele, 1872-1880 : Storia della arte cristiana, t. I-VI, Prato, Gaetano Guasti e Giaghetti e Filio.

Gorman, Michael M., 2003 : «The earliest Latin Commentary on the Gospels », Augustinianum 43/2, p. 253-312, 12 pl.

GraBAR, André, 1966 : L'âge d'or de Justinien. De la mort de Théodose à l'Islam (L'Univers des Formes), Paris, Gallimard.

GregORY, Caspar René, 1900-1909: Textkritik des Neuen Testaments, Leipzig, J.C. Hinrichs'sche Buchhandlung.

GRIESSER, B., 1937 : «Die handschriftliche Ueberlieferung der Expositio IV Evangeliorum des Ps. Hieronymus », Revue bénédictine 49, p. 271-321.

GRYSON, Roger, 1990 : «La version gotique des évangiles. Essai de réévaluation », Revue théologique de Louvain 21, p. 3-31.

- 2000-2003 : Apocalypsis Johannis (Vetus Latina 26/2), ed. Roger GRYsON, Freiburg, Herder.

- 2017 : Victorini Poetoviensis Explanatio in Apocalypsin una cum recensione Hieronymi. Tractatus De fabrica mundi. Fragmentum De vita Christi, cura et studio Roger GRYSON (CC SL 5), Turnhout, Brepols.

GuIGNARD, Christophe, 2017 : «Le quadruple Évangile chez Irénée », dans Irénée de Lyon et les débuts de la Bible chrétienne (Instrumenta Patristica et Mediaevalia, 77), Turnhout, Brepols, p. 101-168.

- 2019 : «The Muratorian Fragment a late Antiquity fake? An answer to C.K. Rothschild», Revue des sciences religieuses 93 1/2, p. 73-90.

HARnACK, Adolf von, 1921 : «Neue Fragmente des Werks des Porphyrius gegen die Christen. Die Pseudo-Polycarpiana und der Schrift des Rhetors Pacatus gegen Porphyrius », dans Sitzungsberichte der Preussischen Akademie der Wissenschaften, Jahrgang 1921, p. 266-284; « Nachträge », p. 834-835.

HeCQUet-Noti, Nicole, 2011 : Avit de Vienne, Éloge consolatoire de la chasteté. Sur la virginité. Introduction, texte critique, traduction, notes et index par Nicole HECQUET-NOTI (Sources chrétiennes 546), Paris, Cerf.

Houghton, Hugh A.G., 2016 : The Latin New Testament. A Guide to its Early History, Texts, and Manuscripts, Oxford, Oxford University Press.

Jullien, Marie-Hélène, Perelman, Françoise, 1994 : Clavis des auteurs latins du moyen âge. Territoire français 735-987. T. I. Turnhout, Brepols.

KER, Neil Ripley, 1957 : Catalogue of Manuscripts containing Anglo-Saxon, Oxford.

LEFORT, Louis Théophile, 1949 : «Fragments de S. Luc en akhmîmique », Le Muséon 62, p. 199-205, pl. IX et X. 
— 1953 : «Fragments bibliques en dialecte akhmîmique », Le Muséon 66, p. 1-30, 2 pl.

MALlon, Alexis, 1906, 1907 : "Une école de savants égyptiens au moyen âge », Mélanges de la Faculté Orientale. Université Saint-Joseph, Beyrouth, 1, p. 109-131 ; 2, p. 213-264.

MerCATI, Giovanni, 1941 : Nuove note di letteratura biblica e cristiana antica (Studi e Testi, 95), Città del Vaticano.

MetZger, Bruce M., $1997^{2}$ : The Canon of the New Testament. Its origin, Development, and Significance, Oxford, Oxford University Press.

Munier, Henri, 1930 : La Scala copte 44 de la Bibliothèque nationale de Paris. Transcription et vocabulaire (Bibliothèque d'études coptes, 2), Le Caire, Institut français d'archéologie orientale.

Petitmengin, Pierre, 2002 : «Chronica Tertullianea et Cyprianea $2001 »$, Revue des Études Augustuniennes 48, p. 331-371.

POwELl, R., 1956 : " The Book of Kells - The Book of Durrow. Comments on the vellum, the make-up and other aspects », Scriptorium 10, p. 3-21.

PREUSCHEN, Erwin, 1893: Analecta, Freiburg i. Br., Leipzig, J.C.B. Mohr.

QuECKE, Hans, 1984: Das Johannesevangelium saïdisch. Text des Handschrift Ppalau Rib.Inv.-Nr. 183 (Papyrologica Castroctaviana, 11), Rome, Barcelone.

REGUL, Jürgen, 1969 : Die antimarcionitischen Evangelienprologe (Aus der Geschichte der lateinischen Bibel, 6), Freiburg, Herder.

SkEAT, Theodore Cressy, 1992 : «Irenäus and the Four-Gospels Canon », Novum Testamentum 34, p. 194-199.

- 1993 : «A codicological analysis of the Chester Beatty papyrus codex of Gospels and Acts», Hermathena 155, p. 27-43.

Soden, Hermann von, 1902 : Die Schriften des Neuen Testaments. T. I, Berlin, Alexander Duncker.

Stone, Michael E., 1976 : Armenian Canon Lists III - The Lists of Mechitar of Ayrevank (c. 1285 C.E.), Harvard Theological Review 69/3-4, p. 289-300.

Stuhlfauth, G., 1942 : «Das Schiff als Symbol der altchristlichen Kunst », Rivista di archeologia cristiana 19, p. 111-141.

SzekUla, Augustin, 1949 : Die Reihenfolge der Bücher des Neuen Testaments bei der Armenier (Nationalbibliothek, 160), Wien, Mechitaristen-Buchdruckerei (en arménien).

TURNER, Cuthbert Hamilton, 1931 : The Oldest Manuscript of the Vulgate Gospels, Oxford, Clarendon Press.

- 1939 : Ecclesiae Occidentalis Monumenta Iuris Antiquissima ... Opus postumum., II, iii, ed. Cuthbertus Hamilton TuRNER, Oxford, Clarendon Press.

VAn Der Meer, Frits, Mohrmann, Christine, 1960 : Atlas de l'Antiquité chrétienne, Paris, Bruxelles, Sequoia.

VAN HAELST, Joseph, 1976 : Catalogue des papyrus littéraires juifs et chrétiens, Paris, CNRS.

Waltz, Pierre, 1928 : Anthologie Palatine I, Paris, Les Belles Lettres. 
Wordsworth, John, SANDAY, William, White, Henry Julian, 1886 : Portions of the Gospels according to St. Mark and St. Matthew (Old Latin Biblical Texts, 2). Oxford, Clarendon Press.

Wormald, Francis, 1954 : The miniatures of the Gospel of St Augustine (Corpus Christi College MS. 286), Cambridge, Cambridge University Press.

Wright, W., 1870 : Catalogue of the Syriac Manuscripts in the British Museum, t. I, London, British Museum.

ZAHN, Theodor, 1883 : Forschungen zur Geschichte des neutestamentlichen Kanons und der altkirchlichen Literatur. II Teil. Der Evangeliencommentar des Theophilus von Antiochien, Erlangen, Andreas Deichert.

- 1890, 1892: Geschichte des neutestamentlichen Kanons. Zweiter Band. Urkunden und Belege zum ersten und dritten Band, Erlangen, Deichert'sche Verlagbuchhandlung.

- 1893 : Forschungen zur Geschichte des neutestamentlichen Kanons. V. Teil, Erlangen, Deichert'sche Verlagbuchhandlung.

\section{RÉSUMÉ}

L'ordre usuel des Évangiles s'est généralisé progressivement depuis la deuxième moitié du $\mathrm{IV}^{\mathrm{e}}$ siècle, suite au choix des grands scriptoria grecs (Alexandrie, Césarée, Antioche, Constantinople); il s'est introduit dans le monde latin par la traduction de Jérôme à la fin du $\mathrm{IV}^{\mathrm{e}}$ siècle. Après Theodor Zahn (1892), Bruce M. Metzger (1997) et une première mise à jour (1999), il est possible aujourd'hui de compléter substantiellement la liste des témoins de treize ordres différents attestés par des manuscrits des Évangiles, par des commentaires des Évangiles, par les listes des livres canoniques, ou encore par des mentions occasionnelles. L'histoire la plus ancienne est conditionnée par les progrès dans la fabrication du codex, permettant la copie d'un, puis de deux, de trois, de quatre Évangiles dans un même codex, et finalement de toute la Bible dans d'épais pandectes. Des considérations chronologiques et autres ont pu guider le choix de l'ordre. La multiplicité des ordres est particulièrement sensible dans le domaine latin, largement tributaire de manuscrits grecs moins bien conservés ou perdus; elle est aussi perceptible dans d'autres versions anciennes (syriaque, copte, arménien, gotique).

\section{ABSTRACT}

The standard sequence of the Gospels spread progressively since the second half of the fourth century, following the choice of the great Greek scriptoria (Alexandria, Caesarea, Antiochia, Constantinople); it enters the Latin area through Jerome's translation at the end of the fourth century. After Theodor Zahn (1892), Bruce M. Metzger (1997), and a first updating (1999), it is possible today to complete significantly the list of the witnesses of thirteen various sequences recorded in manuscripts of the Gospels, in commentaries of the Gospels, in lists of canonical books of the Bible, and in occasional mentions. The earlier history is determined by progress in the manufacturing of the codex, which allowed to copy, one, then two, three, four Gospels in one codex and, at last, the complete Bible in thick pandectes. Chronological and other thoughts could have guided the choice of the sequences. Their multiplicity is especially perceptible in the Latin area, heavily dependent on Greek manuscripts, badly preserved or lost. This multiplicity is also perceptible in other early versions (Syriac, Coptic, Armenian, Gothic). 


\section{MOTS-CLEFS}

1. Évangiles

2. Listes canoniques

3. Codex

4. Vetus Latina

5. Versions orientales

\section{KEYWORDS}

1. Gospels

2. Canon lists

3. Codex

4. Old Latin

5. Oriental versions 